\title{
Corrigendum: In vivo genome editing and organoid transplantation models of colorectal cancer and metastasis
}

Jatin Roper, Tuomas Tammela, Naniye Malli Cetinbas, Adam Akkad, Ali Roghanian, Steffen Rickelt, Mohammad Almeqdadi, Katherine Wu, Matthias A Oberli, Francisco Sánchez-Rivera, Yoona K Park, Xu Liang, George Eng, Martin S Taylor, Roxana Azimi, Dmitriy Kedrin, Rachit Neupane, Semir Beyaz, Ewa T Sicinska, Yvelisse Suarez, James Yoo, Lillian Chen, Lawrence Zukerberg, Pekka Katajisto, Vikram Deshpande, Adam J Bass, Philip N Tsichlis, Jacqueline Lees, Robert Langer, Richard O Hynes, Jianzhu Chen, Arjun Bhutkar, Tyler Jacks \& Ömer H Yilmaz

Nat. Biotechnol. 35, 569-576 (2017); published online 1 May 2017; corrected after print 6 July 2017

In the version of this article initially published, the initial “J” was omitted from an author's name, which should appear as Francisco J Sánchez-Rivera. The error has been corrected in the HTML and PDF versions of the article.

\section{Corrigendum: Shotgun metagenomics, from sampling to analysis}

Christopher Quince, Alan W Walker, Jared T Simpson, Nicholas J Loman \& Nicola Segata

Nat. Biotechnol. 35, 833-844 (2017); published online 12 September 2017; corrected after print 12 September 2017

In the version of this article initially published, the Competing Financial Interests should have indicated the authors had competing interests, but instead indicated there were none. The detailed statement was missing from the HTML: J.T.S. receives research funding from Oxford Nanopore Technologies and has received travel and accommodations to speak at meetings hosted by Oxford Nanopore Technologies. N.J.L. has received honoraria to speak at Oxford Nanopore and Illumina meetings, and travel and accommodation to attend company-sponsored meetings. N.J.L. has ongoing research collaborations with Oxford Nanopore who have provided free-of-charge sequencing reagents as part of the MinION Access Programme and directly in support of research projects. In addition, the publication date was given as 11 September, rather than 12 September 2017. The errors have been corrected for the PDF and HTML versions of this article.

Corrigendum: Development and function of human innate immune cells in a humanized mouse model

Anthony Rongvaux, Tim Willinger, Jan Martinek, Till Strowig, Sofia V Gearty, Lino L Teichmann, Yasuyuki Saito, Florentina Marches, Stephanie Halene, A Karolina Palucka, Markus G Manz \& Richard A Flavell

Nat. Biotechnol. 32, 364-372 (2014); published online 16 March 2014; corrected after print 8 November 2017

In the version of this article initially published, the cells labeled Me290 were Me275 cells. Both Me275 and Me290 are human metastatic HLA-A201+ melanoma cell lines, and both of them were obtained from the Ludwig Cancer Institute. Therefore, the mislabeling does not affect the conclusions of the paper. The error has been corrected in the HTML and PDF versions of the article.

\section{Erratum: Revisiting the Warnock rule}

J Benjamin Hurlbut, Insoo Hyun, Aaron D Levine, Robin Lovell-Badge, Jeantine E Lunshof, Kirstin R W Matthews, Peter Mills, Alison Murdoch, Martin F Pera, Christopher Thomas Scott, Juliet Tizzard, Mary Warnock, Magdalena Zernicka-Goetz, Qi Zhou \& Laurie Zoloth

Nat. Biotechnol. 35, 1029-1042 (2017); published online 9 November 2017; corrected after print 10 November 2017

In the version of this article initially published, J.E. Lunshof's affiliation was given as “Center for Bioethics, Harvard Medical School, Boston, Massachusetts, USA"; the affiliation should have included her main affiliation at the University of Groningen and read, "Department of Genetics, University of Groningen, University Medical Center Groningen (UMCG), Groningen, The Netherlands, and Department of Genetics, Church laboratory, Harvard Medical School, Boston, Massachusetts, USA." In addition, "Divinity School, University of Chicago," should be "University of Chicago Divinity School." The errors have been corrected in the HTML and PDF versions of the article. 